\title{
Tachycardia and Pre-existing Chronic Kidney Disease Are Predictors of the Worse Clinical Outcomes in Patients Recently Hospitalized With Acute Heart Failure
}

Leonardo P. Suciadi ${ }^{1}$, Kevin Wibawa ${ }^{2}$, Giovanni Jessica ${ }^{2}$, Joshua Henrina ${ }^{2}$, Irvan Cahyadi ${ }^{2}$, Bryany T. Santi $^{3}$, Titus K. Hariadi ${ }^{1}$, Firman Tedjasukmana ${ }^{1}$, Nathania M. Kristanti ${ }^{1}$, Elisa F. Pakpahan ${ }^{1}$, Reynold A. Manullang ${ }^{1}$, Antono Sutandar ${ }^{1}$

1. Cardiology, Siloam Heart Institute/Siloam Hospitals Kebon Jeruk, Jakarta, IDN 2. Research, Siloam Heart Institute, Jakarta, IDN 3. Epidemiology, School of Medicine and Health Sciences Atma Jaya Catholic University of Indonesia, Jakarta, IDN

Corresponding author: Leonardo P. Suciadi, be.bakerstreet@gmail.com

\begin{abstract}
Background: This study aimed to assess the factors contributing to the outcomes of recently hospitalized patients with heart failure (HF).
\end{abstract}

Methods: A prospective data of 76 adults who were admitted due to acute HF between October 1, 2019 and June 30, 2020 at our center were analyzed. Endpoints included survival and rehospitalization within six months after discharge.

Results: The mean age was $64.9 \pm 13.8$ years, with a male preponderance (68.4\%). Approximately $60.5 \%$ of patients had the left ventricular ejection fraction (LVEF) $<40 \%$, whereas $26.3 \%$ of patients had LVEF $\geqslant 50 \%$. Coronary artery disease (75\%), arterial hypertension (72.4\%), chronic kidney disease (46.1\%), and diabetes mellitus (46.1\%) were the most frequent comorbidities. Poor compliance $(40.8 \%)$ and non-cardiac infection (21.1\%) were the common precipitating factors for hospitalization. The majority of subjects had severe symptoms, indicated by the frequent need of intensive care unit (43\%), high $\mathrm{N}$-terminal prohormone brain natriuretic peptide levels [NT-proBNP; median, 4765 (1539.7-11782.2) pg/mL], and presence of either atrial fibrillation, severe mitral regurgitation, or significant pulmonary hypertension in approximately one-third of cases. Even though in-hospital mortality was relatively low (2.6\%), the all-cause mortality and rehospitalization rates in the next six months after discharge were still high, reaching $22.54 \%$ and $19.72 \%$, respectively. Further survival analysis showed that tachycardia on admission and pre-existing chronic kidney disease (CKD) resulted in low six-month survival rates among these patients.

Review began 06/02/2021 Review ended 06/11/2021 Published 06/21/2021

\section{(c) Copyright 2021}

Suciadi et al. This is an open access article distributed under the terms of the Creative Commons Attribution License CC-BY 4.0., which permits unrestricted use, distribution, and reproduction in any medium, provided the original author and source are credited.
Conclusion: After hospital discharge, patients with HF were still exposed to higher risks of death and readmission albeit with the medication addressed. Tachycardia on admission and pre-existing CKD might predict worse outcomes.

\section{Categories: Cardiology}

Keywords: heart failure, mortality, rehospitalization, tachycardia, chronic kidney disease

\section{Introduction}

Heart failure (HF) is a major global problem and the leading cause of morbidity and mortality worldwide [1]. HF is the primary reason for hospitalization in the elderly population, and the number of readmissions is high in this population at risk [2]. These data showed that HF is a crucial issue and challenge for health services in the community. Acute HF (AHF) refers to the rapid onset or worsening of symptoms and/or signs of $\mathrm{HF}$, which require prompt treatment and frequently related to unplanned hospital admission or emergency room visit [3]. Generally, AHF encompasses a complex syndrome, including various clinical presentations, underlying cardiac problems, trigger factors, comorbidities, and complications, leading to a relatively high mortality rate during hospitalization and after discharge [4]. Therefore, patient-based, rather than disease-based, data might provide essential information to evaluate miscellaneous aspects of hospitalized patients with AHF [5,6]. Consequently, this approach could improve the clinical assessment and management strategy to reduce in-hospital mortality and rehospitalization rates caused by AHF.

To date, there are numerous published data on the epidemiology, clinical management, and outcomes of AHF in Western countries. However, similar information is still lacking and less comprehensive in Southeast Asian countries. Multiple factors, including different cultures, less organized systems, limited access to healthcare, poor adherence of patients, limited diagnostic or therapeutic tools, and lack of trained investigators, affect the scarce data provided in most Asian countries, including Indonesia [6,7]. Moreover, this lack of data is a major barrier to participation in global collaboration of the relevant issue [8]. Notably, 
several published registries of AHF in Asian countries showed that the demographic characteristics, comorbidities, treatment approaches, and outcomes of patients with AHF are slightly different from the Western data $[9,10]$. Thus, it is pivotal to identify any differences to elaborate on whether the Western data and guidelines regarding the management of patients with AHF can be practically applied to Asian patients, especially in Indonesia.

This study aimed to describe the clinical characteristics and analyze the essential factors contributing to outcomes of hospitalized patients with AHF. This study will be of value in adding databases of AHF during hospitalization and until six months after discharge, especially in the Indonesian population. Thus, the results might lead to a protocol to reduce mortality and rehospitalization rates related to AHF.

\section{Materials And Methods}

This prospective study included all patients aged $\geqslant 18$ years who were admitted to our hospital with the primary indication of AHF between October 1, 2019 and June 30, 2020, at Siloam Hospital Kebon Jeruk, Jakarta. AHF was defined based on the criteria delineated in 2016 European Society of Cardiology guidelines for HF [3]. Patients were further categorized to acute de novo HF (admitted for the first time without a history of HF) and acute decompensated HF (ADHF; sudden worsening of HF in previously diagnosed or hospitalized patients). Patients who were discharged or transferred by their own intention before fulfilling treatment or admitted by other primary reasons than AHF were excluded.

Data regarding the patients' demographic, clinical characteristics, comorbidities, history of cardiovascular procedures, HF etiology, precipitating factors, laboratory investigation, electrocardiography, echocardiography, and management during hospitalization were directly collected from hospital medical records. The outcomes were in-hospital mortality, defined as all-cause death during hospitalization for AHF; six-month rehospitalization, defined as rehospitalization for HF within six months after hospital discharge; and six-month mortality, defined as all-cause mortality within six months after hospital discharge. The outcome data regarding in-hospital mortality were collected from the medical records, and data on six months of rehospitalization and mortality were collected by either telephone contact or clinical visit within six months after hospital discharge.

Descriptive statistics were used to summarize the data. Categorical variables were presented as frequencies and percentages. For continuous variables, Shapiro-Wilk or Kolmogorov-Smirnov normality test was performed as appropriate. Normally distributed data were summarized as mean and standard deviation. Otherwise, median and interquartile range (25th and 75 th percentiles) were used. Chi-square test or Fischer's exact test were performed to compare and determine the independent variables according to sixmonth mortality that will be included in the logistic regression model. Independent variables included in the Cox regression model were variables with chi-square or Fisher's exact $\rho$-value $<0.25$. Survival estimates for six-month mortality were calculated using the Kaplan and Meier method. Statistical analysis was conducted using SPSS version 22.0.

\section{Results}

This study included a total of 76 patients admitted with the primary complaint of AHF during a nine-month registry period. The characteristics, comorbidities, and history of cardiovascular procedures are presented in Table 1 . The mean age of the subjects was $64.9 \pm 13.8$ years, and the majority of patients (68.4\%) were male. Notably, nearly one-third of our patients were very elderly with age $\geqslant 75$ years. The median body mass index of the patients was $24.6(22.6-27.3) \mathrm{kg} / \mathrm{m}^{2}$. Moreover, $71 \%$ of subjects were classified as overweight or obese, and only $3.9 \%$ of them were underweight $\left(\mathrm{BMI}<18.5 \mathrm{~kg} / \mathrm{m}^{2}\right)$.

\section{Characteristics}

Age, mean \pm SD (years)

$\geq 75$ years

$<75$ years

Male sex

BMI, $\mathrm{kg} / \mathrm{m}^{2}$, median (IQR)

Underweight

Normal

Overweight

Obese

\section{n (\%)}

$64.96 \pm 13.79$

22 (28.95)

54 (71.05)

52 (68.4)

24.65 (22.6-27.34)

3 (3.95)

$19(25)$

$19(25)$

$35(46.05)$ 


\section{Cureus}

Duration of hospitalization in the cardiac ward, median (IQR), days

Duration of hospitalization in the ICCU, median (IQR), days

Number of patients needing intensive cardiac care

Smoking habit

Active smoker

Ex-smoker

Never smoke

Comorbidity

Patients with cardiovascular comorbidity

Patients with non-cardiovascular comorbidity

Patients with both cardiovascular and non-cardiovascular comorbidities

CAD

Hypertension

55 (72.4)

CKD without hemodialysis

Non-insulin-dependent DM

Obesity

Other comorbidities

Persistent/permanent atrial fibrillation

Other systemic diseases (autoimmune, chronic inflammatory, connective tissue)

Insulin-dependent DM

Geriatry and frailty

Stroke/TIA

Chronic liver disease

CKD on hemodialysis

COPD

Chronic anemia

Cachexia

Cancer

History of cardiovascular procedure

$\mathrm{PCl}$

CABG

Heart valve repair/replacement

Other cardiovascular procedures (angiography)

CRT

\section{TABLE 1: Basic characteristic of the study cohort.}

$\mathrm{BMI}$, body mass index; CABG, coronary artery bypass graft; CAD, coronary artery disease; CKD, chronic kidney disease; COPD, chronic obstructive pulmonary disease; CRT, cardiac resynchronization therapy; DM, diabetes mellitus; ICCU, intensive cardiac care unit; IQR, interquartile range; PCI, percutaneous coronary intervention; SD, standard deviation; TIA, transient ischemic attack. 


\section{Cureus}

hospitalization in the cardiac ward and intensive care unit was 5 (4-9) days and 4 (2.5-7) days, respectively. The common comorbidities observed among our patients were coronary artery disease (CAD) (75\%), arterial hypertension (72.4\%), chronic kidney disease (CKD) without hemodialysis (46.1\%), and non-insulindependent diabetes mellitus (46.1\%). Some patients had a history of undergoing previous cardiovascular procedures including percutaneous coronary intervention (18.4\%), coronary artery bypass grafting (14.5\%), surgical valve repair/replacement (5.3\%), and cardiac resynchronization therapy (1.3\%).

Most patients (65.8\%) had systolic blood pressure between 90 and $139 \mathrm{mmHg}$ at the time of admission, whereas the median heart rate was 88 beats per minute (bpm) (Table 2). Underlying CAD was the most common etiology for AHF, encompassing chronic coronary syndrome (50\% cases), prior myocardial infarction (17.1\%), and acute coronary syndrome (15.8\%). Based on the time interval of the event, only $13.2 \%$ of patients had de novo HF, whereas the remaining patients had ADHF. Meanwhile, with respect to the clinical profiles, the majority of patients (88.2\%) presented with ADHF, and only $10.5 \%$ and $1.3 \%$ of them had acute lung edema with respiratory distress and cardiogenic shock, respectively. In patients with $\mathrm{ADHF}$, the common precipitating factors were poor compliance (40.8\%), non-cardiac infection (21.1\%), acute coronary syndrome $(9.2 \%)$, hypertensive emergency (3.9\%), and acute mechanical cause (2.6\%).

Variables

Clinical presentation

SBP at admission, $\mathrm{mmHg}$, median (IQR)

SBP at admission $<90 \mathrm{mmHg}$

SBP at admission $90-139 \mathrm{mmHg}$

SBP at admission $\geq 140 \mathrm{mmHg}$

$\mathrm{DBP}$ at admission, $\mathrm{mmHg}$, mean $\pm \mathrm{SD}$

HR at admission, bpm, median (IQR)

Raised JVP

Lung crepitations

Leg edema

Ascites

Pleural effusion

Cyanosis

Etiology

Chronic coronary syndrome

Old myocardial infarction with scar tissue

VHD

Mitral stenosis

Mitral regurgitation

Aortic stenosis

Aortic regurgitation

Etiology of VHD

Infective endocarditis

Degenerative

Rheumatic heart disease

Congenital bicuspid/prolapse

Other etiologies of VHD

$5(6.6)$

ACS 


\section{Cureus}

Hypertensive heart disease

12 (15.8)

Idiopathic/familial DCM

Metabolic/nutritional

Other etiologies

AHF onset

De novo

ADHF from chronic HF

AHF precipitating factor

Poor compliance

Non-cardiac infection

ACS

Hypertensive emergency

Acute mechanical cause

Others (high blood sugar, suboptimal drug dose)

AHF clinical profile

ADHF

Acute lung edema (respiratory distress)

Cardiogenic shock

$1(1.3)$

\section{TABLE 2: Clinical presentation, etiology, onset, precipitating factor, and AHF clinical profile of} study cohort.

ACS, acute coronary syndrome; ADHF, acute decompensated heart failure; AHF, acute heart failure; bpm, beats per minute; DBP, diastolic blood pressure; DCM, dilated cardiomyopathy; HF, heart failure; HR, heart rate; IQR, interquartile range; JVP, jugular venous pressure; SBP, systolic blood pressure; SD, standard deviation; VHD, valvular heart disease.

As shown in Table 3, the mean hemoglobin level at admission was $12.51 \pm 2.3 \mathrm{mg} / \mathrm{dL}$. Most patients had renal impairment with a median creatinine level of $1.7(1.2-2.6) \mathrm{mg} / \mathrm{dL}$, indicating a median estimated glomerular filtration rate of 38 (22.9-59.6) mL/min. Unfortunately, only a few patients (31.6\%) underwent $\mathrm{N}$-terminal prohormone brain natriuretic peptide (NT-proBNP) tests, and the median level of this cardiac biomarker at the time of admission was 4765 (1539.7-11782.2) pg/mL. Atrial fibrillation was identified in $29 \%$ of initial electrocardiography (ECG) of our patients. Widened QRS complex $\geqslant 120 \mathrm{~ms}$ on ECG was found in $13.3 \%$ of subjects. The premature ventricular complex was common in the ECG result, which was noted in $13.3 \%$ of patients.

\section{Variable}

Laboratory results, median (IQR), unless specified otherwise

Hemoglobin, mean \pm SD, g/dL

RDW, mean $\pm S D, \%(n=3)$

Serum iron, mean $\pm S D, \mu g / d L ~(n=14)$

TIBC, $\mu \mathrm{g} / \mathrm{dL},(\mathrm{n}=14)$

TSAT, mean \pm SD, $\%(n=14)$

Ferritin, $n g / m L, ~(n=14)$

$\mathrm{Cr}, \mathrm{mg} / \mathrm{dL}$

eGFR, $\mathrm{mL} / \mathrm{min}$
Value

\author{
$12.51 \pm 2.3$ \\ $16.7 \pm 4.91$ \\ $62.71 \pm 54.72$ \\ 201 (193.25-238.25) \\ $40.26 \pm 26.52$ \\ $279.11(126.4-526.14)$ \\ $1.7(1.2-2.58)$
}

$38.06(22.94-59.64)$ 


\section{Cureus}

Sodium, mEq/L

Potassium, mEq/L

Total bilirubin, mg/dL $(\mathrm{n}=17)$

Albumin, mean $\pm \mathrm{SD}, \mathrm{mg} / \mathrm{dL}(\mathrm{n}=21)$

NT-proBNP, pg/mL ( $n=24)$

Troponin T

Troponin T-test, n (\%)

Increased value of troponin T-test, n (\%)

SGOT, U/L ( $n=59)$

SGPT, U/L ( $=59)$

ECG, n (\%), unless specified otherwise

Rhythm

Sinus

Atrial fibrillation

Paced

Other rhythms

HR, bpm, median (IQR) ( $=75)$

QRS duration, ms, median (IQR) $(n=75)$

QRS duration $<120 \mathrm{~ms}$

QRS duration $\geq 120 \mathrm{~ms}$

QTc duration, msec, median (IQR) $(\mathrm{n}=75)$

QTc duration $\leq 460 \mathrm{~ms}$

QTc duration $>460 \mathrm{~ms}$

$\operatorname{PVC}(\mathrm{n}=75)$

Without

Infrequent

Frequent

Echocardiography, n (\%) unless specified otherwise

LA diameter $(n=50)$

$\geq 40 \mathrm{~mm}$

$<40 \mathrm{~mm}$

LAVi, $\mathrm{mL} / \mathrm{m}^{2}$, mean $\pm \mathrm{SD}(\mathrm{n}=11)$

LV EDD, mm, mean \pm SD $(n=36)$

E/e', median (IQR) $(n=37)$

LVEF

$\geq 50 \%$

$40-49 \%$

$<40 \%$

Diastolic dysfunction $(n=53)$
$138(135-140)$

$3.9(3.43-4.5)$

$1.63(0.99-5.05)$

$3.24 \pm 0.66$

4765 (1539.75-11782.25)

$22(28.9 \%)$

$13(59.1 \%)$

29 (19-49)

22 (16-35)

$51(67.1)$

22 (29)

1 (1.3)

2 (2.6)

94 (79-119)

$80(80-100)$

$65(86.7)$

$10(13.3)$

437 (400-460)

58 (77.3)

17 (22.7)

65 (86.7)

$6(8)$

4 (5.3)

$40(80.0)$

$10(20.0)$

$58.91 \pm 24.36$

$55.13 \pm 8.953$

19.00 (12.75-23.00)

20 (26.3)

10 (13.2)

$46(60.5)$

48 (90.57) 


\section{Cureus}

No diastolic dysfunction

Unable to evaluate

Grading of diastolic dysfunction $(n=48)$

Grade I

Grade II

Grade III

Mitral regurgitation $(n=59)$

Severe

Mild-moderate

No mitral regurgitation

PASP $(n=46)$

$\geq 50 \mathrm{mmHg}$ (high likelihood)

35-50 $\mathrm{mmHg}$ (intermediate likelihood)

$<35 \mathrm{mmHg}$ (low likelihood)

TAPSE $(n=47)$

$<17 \mathrm{~mm}$

$\geq 17 \mathrm{~mm}$

RV base size $(n=45)$

$\geq 42 \mathrm{~mm}$

$<42 \mathrm{~mm}$

$31(68.89)$

TABLE 3: Laboratory, electrocardiographic, and echocardiographic results of the study cohort.

bpm, beats per minute; Cr, creatinine; ECG, electrocardiography; eGFR, estimated glomerular filtration rate; HR, heart rate; IQR, interquartile range; LA, left atrium; LAVi, left atrial volume index; LV, left ventricle; LVEF, left ventricular ejection fraction; NT-proBNP, N-terminal prohormone brain natriuretic peptide; PASP, pulmonary artery systolic pressure; PVC, premature ventricular complex; RDW, red cell distribution width; RV, right ventricle; SD, standard deviation; SGOT, serum glutamic oxaloacetic transaminase; SGPT, serum glutamic pyruvate transaminase; TAPSE, tricuspid annular plane systolic excursion; TIBC, total iron-binding capacity; TSAT, transferrin saturation.

By echocardiography study, the left ventricular ejection fraction (LVEF) $<40 \%$ was found in $60.5 \%$ of patients, whereas LVEF $\geqslant 50 \%$ in $26.3 \%$ of subjects. The LVEF was measured using the standard of Simpson's procedure from the apical four-chamber view. Since most patients had diastolic dysfunction, restrictive diastolic dysfunction was noted in $41.7 \%$ of patients. Nearly three-quarters of patients had mitral regurgitation, and severe regurgitation was identified in $27.1 \%$ of cases. More than one-third of subjects had estimated pulmonary artery systolic pressures $>50 \mathrm{mmHg}$, indicating a high likelihood of pulmonary hypertension. The right ventricular dysfunction as simply indicated by reduced tricuspid annular plane systolic excursion was found in $40.4 \%$ of subjects.

During hospitalization, serious ventricular arrhythmias (ventricular tachycardia/fibrillation) occurred in $6.6 \%$ of patients. However, electrical cardioversion was only needed in one patient with unstable hemodynamics during prolonged ventricular tachycardia, as shown in Table 4. Some corresponding procedures required included noninvasive positive pressure ventilation (9.2\%), invasive mechanical ventilation (6.6\%), hemodialysis or ultrafiltration (6.6\%), and insertion of the intra-aortic balloon counterpulsation $(2.6 \%)$.

\section{Variables}

Complication during admission

Respiratory distress and respiratory failure 


\section{Cureus}

\begin{tabular}{|c|c|}
\hline Cardiogenic shock & - \\
\hline Ventricular tachyarrhythmia (VT, VF, TdP) & $5(6.6)$ \\
\hline Atrial fibrillation > $150 \mathrm{bpm}$ & - \\
\hline Cardiac arrest & - \\
\hline Non-cardiac complication & $5(6.6)$ \\
\hline \multicolumn{2}{|l|}{ Injection drugs administered } \\
\hline Furosemide or other diuretics & $70(92.1)$ \\
\hline Nitrate & $33(43.4)$ \\
\hline Dobutamine & $12(15.8)$ \\
\hline Dopamine & $2(2.6)$ \\
\hline Norepinephrine & $8(10.5)$ \\
\hline Milrinone & - \\
\hline Anticoagulant & $16(21.1)$ \\
\hline \multicolumn{2}{|l|}{ Blood product } \\
\hline Whole blood & - \\
\hline Packed red cells & $7(9.2)$ \\
\hline Volume, $\mathrm{mL}$, mean \pm SD & $537.67 \pm 364$ \\
\hline Fresh frozen plasma, mean \pm SD volume, $\mathrm{mL}$ & $3(3.9)$ \\
\hline Volume, $\mathrm{mL}$, mean $\pm \mathrm{SD}$ & $834 \pm 364$ \\
\hline Amiodarone & $4(5.3)$ \\
\hline Digoxin & 7 (9.2) \\
\hline Lidocaine & $3(3.9)$ \\
\hline Antibiotic & $25(32.9)$ \\
\hline Iron supplementation & $1(1.3)$ \\
\hline \multicolumn{2}{|l|}{ Cardiac procedures, during admission } \\
\hline Percutaneous coronary intervention & $4(5.3)$ \\
\hline Percutaneous structural intervention & $1(1.3)$ \\
\hline CABG & $3(3.9)$ \\
\hline Open valve surgery & $2(2.6)$ \\
\hline IABP & $2(2.6)$ \\
\hline CRT implantation & - \\
\hline ICD implantation & - \\
\hline Electrical cardioversion/defibrillation shock & $1(1.3)$ \\
\hline Intubation and mechanical ventilation & $5(6.6)$ \\
\hline Duration of mechanical ventilation, days, mean $\pm \mathrm{SD}$ & $4.83 \pm 4.4$ \\
\hline BiPAP/CPAP & 7 (9.2) \\
\hline Hemodialysis/ultrafiltration & $5(6.6)$ \\
\hline CPR & - \\
\hline Others & $12(15.8)$ \\
\hline
\end{tabular}




\section{Cureus}

\section{TABLE 4: In-hospital management of the study cohort.}

bpm, beat per minute; BiPAP, bi-level positive airway pressure; CABG, coronary artery bypass graft; CPAP, continuous positive airway pressure; CPR, cardiopulmonary resuscitation; CRT, cardiac resynchronization therapy; IABP, intra-aortic balloon pump; ICD, implantable cardioverterdefibrillator; SD, standard deviation; TdP, torsades des pointes; VF, ventricular fibrillation; VT, ventricular tachycardia.

Regarding the management of patients, loop diuretics and nitrates were the most common intravenous drugs used, which contributed to $92.1 \%$ and $43.4 \%$ of patients, respectively. Additionally, inotropes were needed in approximately one-third of the subjects, with dobutamine and norepinephrine as the most preferred agents. Only $21.1 \%$ of patients received parenteral anticoagulation for venous thromboembolism treatment or prophylaxis.

Importantly, the negative fluid balance was achieved in almost all patients (93\%) during hospital stay (Table 5). Table 6 showed the common medications prescribed at discharge included diuretics (77.6\%),

spironolactone (59.2\%), angiotensin-converting enzyme (ACE) inhibitors/angiotensin receptor blocker (ARB) (56.6\%), and beta-blockers (44.7\%). Meanwhile, other familiar HF drugs, such as digoxin, sacubitril/valsartan, ivabradine, and SGLT2 inhibitors were only prescribed in $\leqslant 10 \%$.

\section{Variables}

SBP, $\mathrm{mmHg}(\mathrm{n}=74)$

DBP, $\mathrm{mmHg}$, median (IQR) $(\mathrm{n}=74)$

Heart rate, beats per minute $(n=74)$

Body weight, kg ( $n=69)$

Body water balance, $n(\%)(n=73)$

Positive

Negative

Creatinine, $\mathrm{mg} / \mathrm{dL}$

eGFR, $\mathrm{mL} / \mathrm{min}$

Sodium, $\mathrm{mEq} / \mathrm{L}$, mean $\pm \mathrm{SD}$

Potassium, mEq/L

SGOT, U/L ( $=60)$

SGPT, U/L $(n=60)$

Total bilirubin, $\mathrm{mg} / \mathrm{dL}(\mathrm{n}=18)$

Hemoglobin, g/dL
Median (IQR)

114 (104.5-131.0)

$68.50(59.0-75.25)$

$78.00(70.00-89.25)$

$63.00(54.15-70.00)$

$5(6.84)$

68 (93.16)

$1.55(1.10-2.40)$

$42.61(24.33-69.94)$

$137.75 \pm 3.36$

$3.80(3.52-4.1)$

$29.00(19.25-52.00)$

$22.50(16.25-36.50)$

$1.83(1.19-4.70)$

11.90 (10.55-13.87)

\section{TABLE 5: Predischarge condition of the study cohort.}

eGFR, estimated glomerular filtration rate; HR, heart rate; IQR, interquartile range; SD, standard deviation; SGOT, serum glutamic oxaloacetic.

\begin{tabular}{|l|c|}
\hline Medication & N (\%) \\
\hline Furosemide & $57(75)$ \\
$20 \mathrm{mg}$ & $7(9.2)$ \\
$40 \mathrm{mg}$ & 27 (35.5) \\
$60-80 \mathrm{mg}$ & $14(18.5)$ \\
$>80 \mathrm{mg}$ & $9(11.8)$ \\
Hydrochlorothiazide or indapamide & $2(2.6)$
\end{tabular}




\section{Cureus}

\begin{tabular}{|c|c|}
\hline Nitrate & 25 (32.9) \\
\hline $2.5 \mathrm{mg}$ & $1(1.3)$ \\
\hline $5 \mathrm{mg}$ & $4(5.3)$ \\
\hline $10 \mathrm{mg}$ & $2(2.6)$ \\
\hline $15 \mathrm{mg}$ & $6(7.9)$ \\
\hline$>15 \mathrm{mg}$ & $12(15.8)$ \\
\hline ACE inhibitor & $10(13.2)$ \\
\hline Angiotensin receptor blockers & $33(43.4)$ \\
\hline Beta-blockers & $34(44.7)$ \\
\hline Spironolactone & $45(59.2)$ \\
\hline $12.5 \mathrm{mg}$ & $3(3.9)$ \\
\hline $25 \mathrm{mg}$ & $19(25.0)$ \\
\hline $50 \mathrm{mg}$ & 14 (18.4) \\
\hline $100 \mathrm{mg}$ & $9(11.8)$ \\
\hline Aspirin & $16(21.1)$ \\
\hline Clopidogrel & $34(44.7)$ \\
\hline Ticagrelor & - \\
\hline Warfarin & $10(13.2)$ \\
\hline NOAC & $6(7.9)$ \\
\hline Digoxin & $9(11.8)$ \\
\hline $0.125 \mathrm{mg}$ & $7(9.2)$ \\
\hline $0.250 \mathrm{mg}$ & $2(2.6)$ \\
\hline Amiodarone & $5(6.6)$ \\
\hline Ivabradine & $6(7.9)$ \\
\hline Sacubitril/valsartan & $6(7.9)$ \\
\hline Tolvaptan & - \\
\hline Statins & 50 (65.8) \\
\hline Insulin & $11(14.5)$ \\
\hline Empaglifozin/dapaglifozin & $4(5.3)$ \\
\hline Dihydropyridine calcium channel blocker & $12(15.8)$ \\
\hline Nondihydropyridine calcium channel blocker & $3(3.9)$ \\
\hline Trimetazidine & $2(2.6)$ \\
\hline
\end{tabular}

TABLE 6: Discharge medication prescribed for the study cohort.

$\mathrm{ACE}$, angiotensin-converting enzyme; NOAC, non-vitamin $\mathrm{K}$ oral anticoagulant.

As shown in Table 7, even though in-hospital mortality was relatively low (2.6\%), the number of all-cause mortality and rehospitalization in the next six months after discharge were still considerably high, reaching $22.54 \%$ and $19.72 \%$, respectively. Cox regression analysis showed that tachycardia at admission (HR 1.98; 95\% CI 0.65-5.77) and pre-existing CKD (HR 2.16; 95\% CI 0.52-9.05) increased the mortality rate compared to patients without these conditions, although these findings were not statistically significant (Table 8 ). Further analysis of these parameters in Kaplan Meier revealed that there were poorer six months survival 


\section{Cureus}

rates in patients with tachycardia on admission (log-rank test 0.06; Figures 1 and 2 ) and underlying CKD (0.018; Figures 3 and 4). Patients with initial tachycardia and previous renal dysfunction had a lower mean survival time compared to those without these conditions (133 days vs 161 days, and 136 vs 169 days, respectively). It is important to note that none of these covariates reached the median survival at six months follow-up.

\begin{tabular}{ll} 
Variables & Total $\mathbf{n}(\%)$ \\
\hline In-hospital mortality & $2(2.6)$ \\
Six-month mortality & $16(22.54)$ \\
Six-month rehospitalization & $14(19.72)$
\end{tabular}

TABLE 7: In-hospital mortality, six-month mortality, and six-month rehospitalization.

\begin{tabular}{|c|c|c|c|}
\hline Variable & HR & $95 \% \mathrm{CI}$ & P-value \\
\hline Hypertension during admission & 1.313 & $0.455-3.795$ & 0.615 \\
\hline Tachycardia during admission & 1.938 & $0.651-5.772$ & 0.235 \\
\hline CKD & 2.165 & $0.518-9.053$ & 0.290 \\
\hline Anemia & 1.308 & $0.395-4.330$ & 0.661 \\
\hline eGFR $<45 \mathrm{~mL} / \mathrm{min}$ & 1.780 & $0.356-8.911$ & 0.483 \\
\hline Hyponatremia during admission & 1.586 & $0.515-4.885$ & 0.422 \\
\hline
\end{tabular}

TABLE 8: Cox regression model of six-month follow-up.

CKD, chronic kidney disease; eGFR, estimated glomerular filtration rate. 


\section{Cureus}

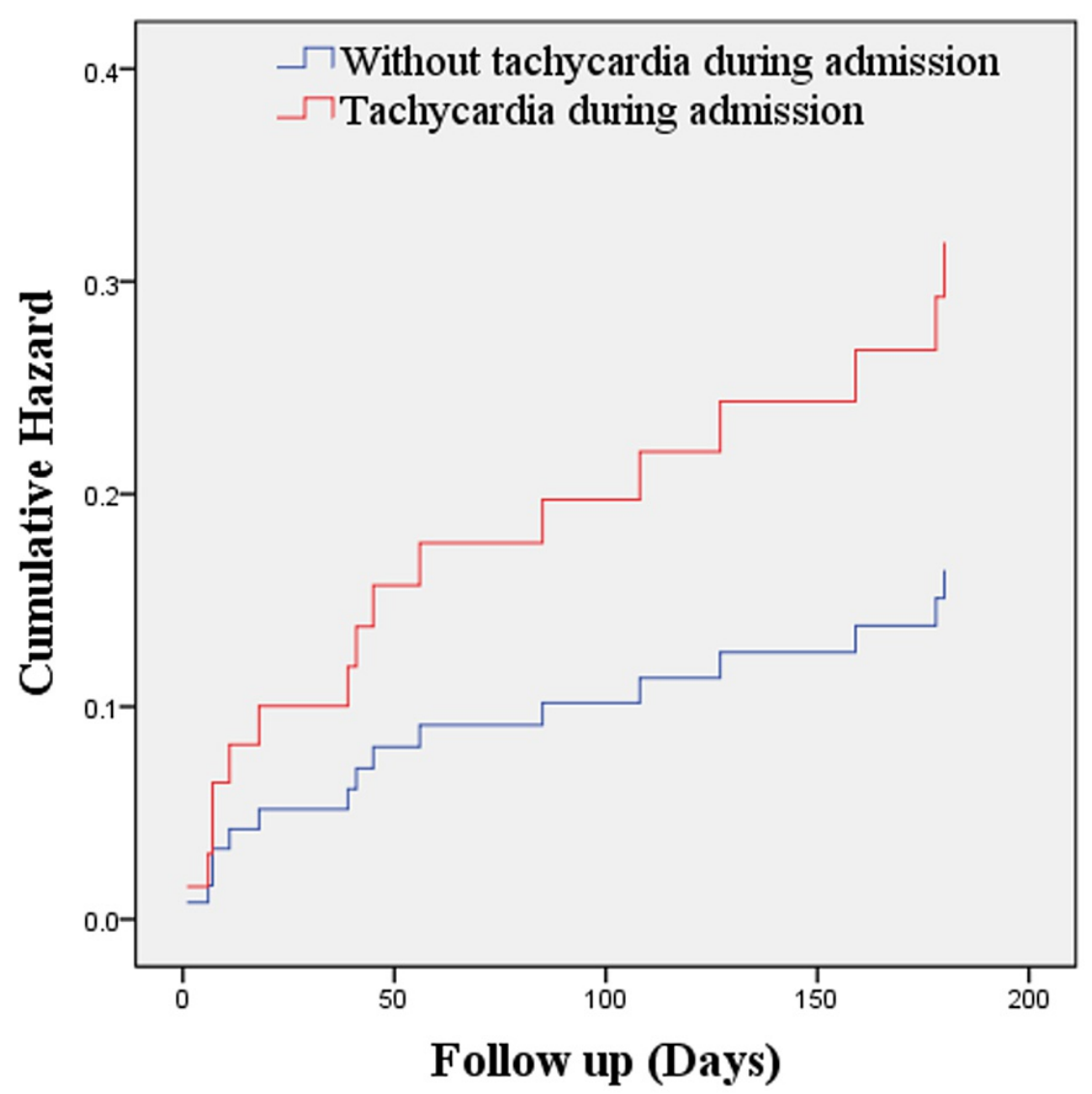

FIGURE 1: Cox regression of six-month mortality of patients with AHF with tachycardia during admission and without tachycardia during admission ( $p=0.235)$.

AHF, acute heart failure. 


\section{Cureus}

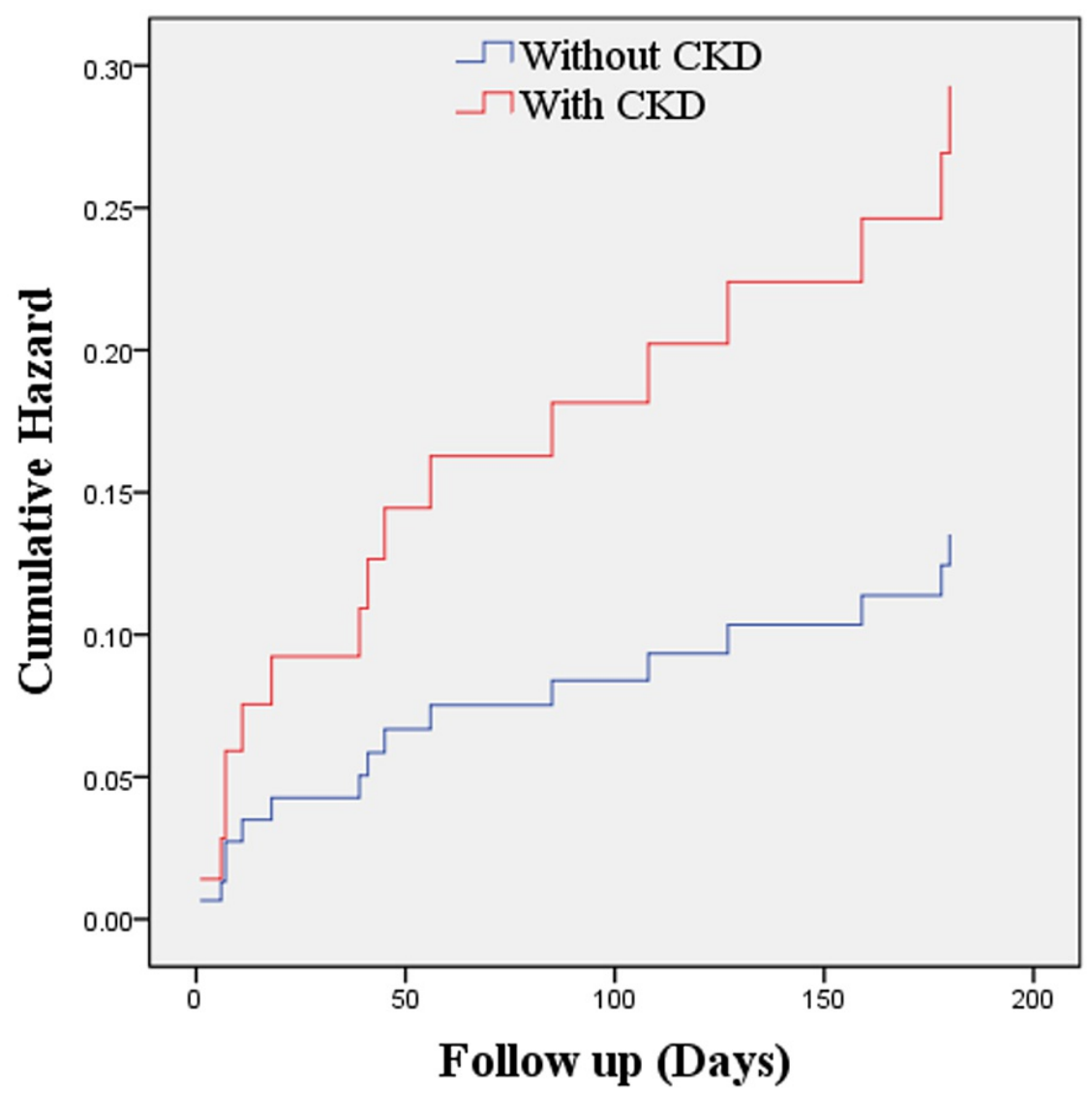

FIGURE 2: Cox regression of six-month mortality of patients with AHF with CKD and without CKD $(p=0.290)$.

AHF, acute heart failure; CKD, chronic kidney disease. 


\section{Cureus}

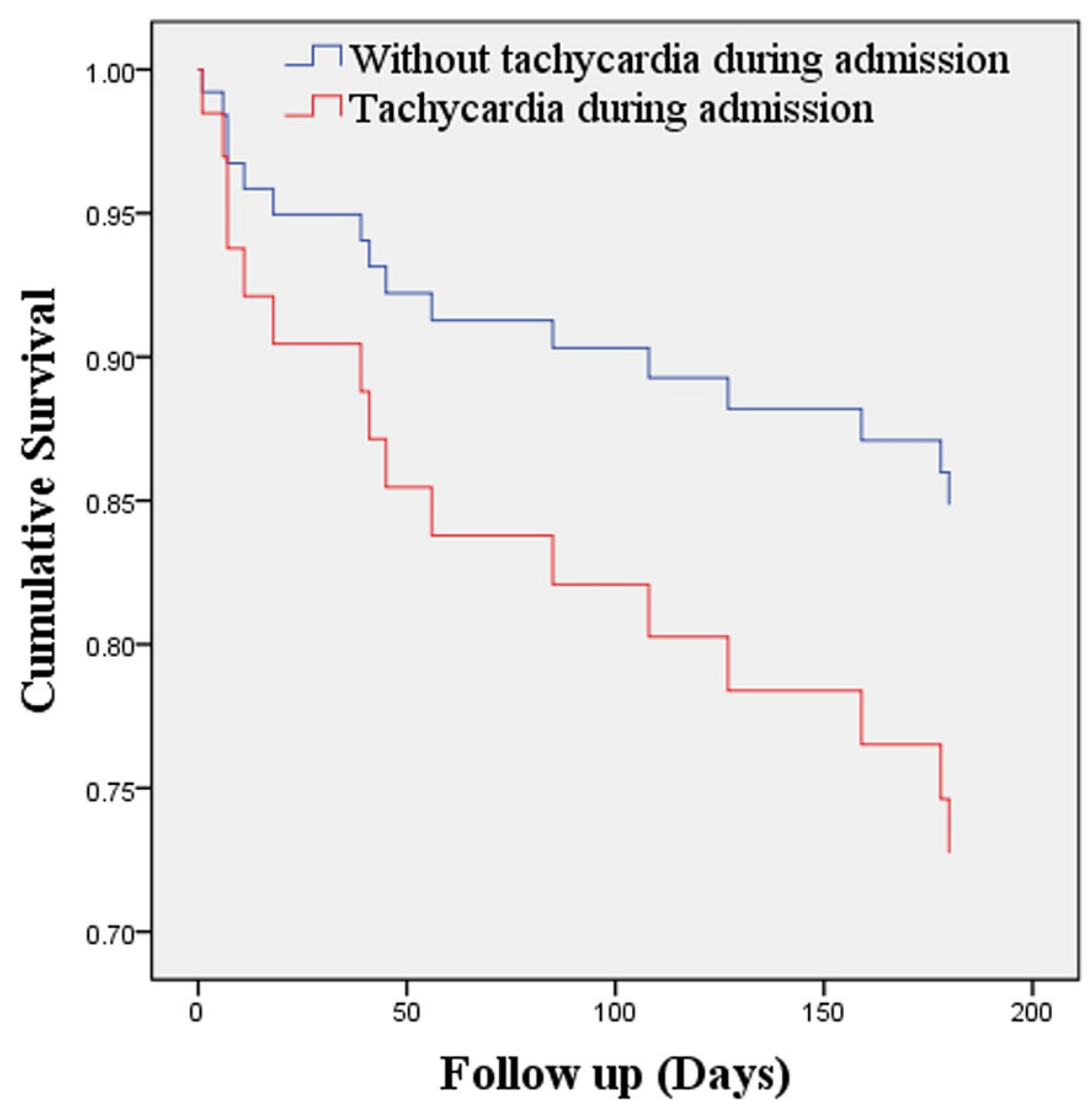

FIGURE 3: Kaplan-Meier estimate of mortality between patients with AHF with tachycardia and without tachycardia during admission (logrank test, 0.06). 


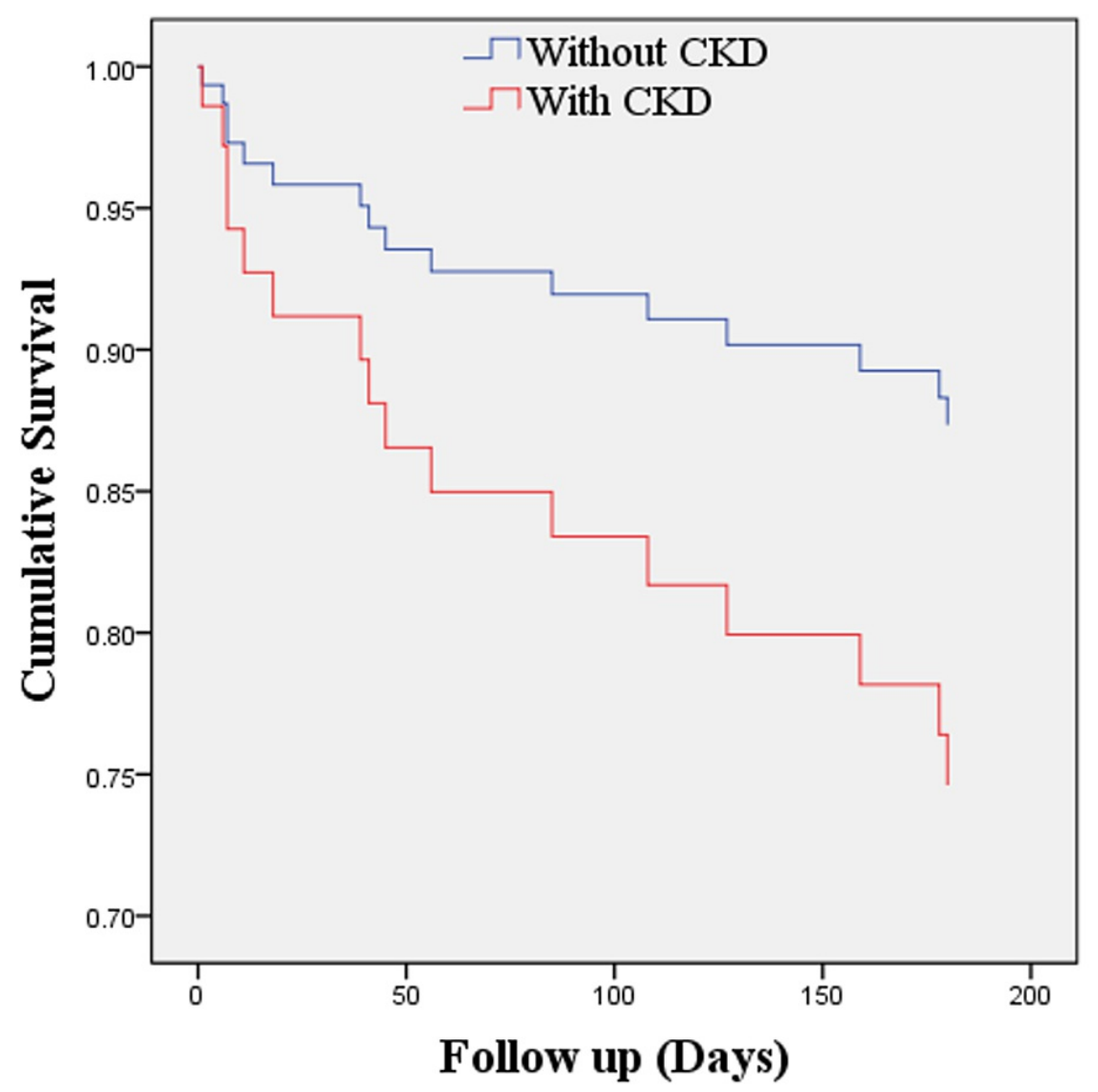

FIGURE 4: Kaplan-Meier estimate of mortality between patients with AHF with CKD and without CKD (log-rank test 0.018).

AHF, acute heart failure; CKD, chronic kidney disease.

\section{Discussion}

The patients in this study were younger (mean age, 64.9 years) than populations in Western studies [11], and were predominantly male and overweight or obese. Specifically, approximately one-third of patients were a very elderly group with age $>75$ years. CAD and hypertension were prevalent comorbidities in patients included in this registry. Interestingly, the majority of HF patients enrolled were overweight and obese. It seems that this overnutrition condition should be considered as an important comorbidity in our population. Additionally, CAD, with various clinical profiles, was the most common etiology of HF in our center and this fact is consistent with the data worldwide [9,11]. Other common causes identified in our population were hypertensive and valvular heart diseases.

At our center, acute decompensation of chronic HF was much more prevalent than the case of de novo counterpart. Importantly, poor compliance of the patients was the commonest precipitating factor of the worsening of HF symptoms, as it was found in nearly half of the admitted patients. This finding delineates the crucial role of patient education and sustainable services after hospital discharge to avoid rehospitalization or mortality. Patients with HF often need to undergo long-term and complex management. Thus, low health literacy may affect patient compliance to HF medication. It is understood that several patients did not understand their current situation and medication, as well as management plan of the disease. This lacking of understanding would easily result in abrupt cessation of the medication soon after the symptoms subsided [12]. Additionally, elderly patients tend to discontinue the medication when adverse drug effects appear [13]. It is important to provide adequate information regarding the common side effects of drugs to the patients. Routine evaluation of drug interaction and polypharmacy could be an effective solution to this problem.

Other frequent precipitants of ADHF in our subjects were non-cardiac infection, and pneumonia was the most common type of infection that we encountered. As generally known, patients with HF had an increased risk of developing pneumonia. Alveolar and interstitial congestion could disrupt physiological mechanisms 
of alveolar lining fluid as the cellular barriers. This damage would impair the microbial clearance and increase the susceptibility of the respiratory tract to infection [14]. Meanwhile, pneumonia and other systemic infections would increase cardiac workload, which is a detrimental situation for patients with chronic HF. The most common bacterial causative agents reported in patients with HF are Streptococcus pneumoniae and Staphylococcus aureus [15]. Besides, various types of viruses, such as influenza, parainfluenza virus, coronavirus, and human metapneumovirus, are also common causes of communityacquired pneumonia in this population. Nevertheless, co-infection by bacteria and viruses often occur $[16,17]$. Regarding this issue, the guideline recommends that patients with HF should receive pneumococcal and yearly influenza vaccination to reduce worsening of symptoms and hospitalization [3]. Besides lung infection, other non-cardiac infections, such as sepsis, urinary tract infection, and even soft tissue infection, can lead to worsening of HF symptoms and hospitalization [18].

Patients with low LVEF ( $<40 \%$ ) dominated in this study ( $60.5 \%$ subjects), and this finding is similar to other Asian registries [9,11]. The higher proportion of HFrEF in our center might be correlated to CAD as the most common etiology and comorbidity encountered here. It is important to note that approximately one-third of patients with HF in this study had either atrial fibrillation, severe functional mitral regurgitation, or significant pulmonary hypertension. The high median NT-proBNP levels $(4765 \mathrm{pg} / \mathrm{mL})$ might indicate the relative severe HF symptoms in our population.

Intravenous diuretic, especially furosemide, was the most commonly administered drug during hospitalization. This agent is effective in a majority of cases of acute HF to relieve the volume overload symptoms, thus gaining negative water balance before discharge [9]. Although diuretic resistance might prohibit decongestion strategy, this problem could be solved by combining some diuretic agents [19]. Intravenous nitrates were also commonly administered to optimize symptom relief at the initial period, as long as there was no hypotension.

The in-hospital mortality rate at our center (2.6\%) was considerably lower compared to the previously reported data from Indonesia, which were $6.7 \%$ and 3\% [7,20]. Despite this lower death rate during hospitalization, the six-month mortality and rehospitalization rates significantly increased to $22.54 \%$ and $19.72 \%$, respectively. Nevertheless, this six-month death rate was still lower than those of the previous reported Asian studies, which were $26.3 \%$ and $45.8 \%$ [21,22]. The relatively high mortality and hospital readmission rates within the next six months after discharge emphasized that $\mathrm{HF}$ is a serious disease with a rapidly progressive condition, albeit proper management during hospitalization. Thus, sustainable optimization of treatment after discharge is of paramount importance to reduce adverse events in the future. Delivering education and improving patients' compliance might offer an effective way to obtain better long-term outcomes; particularly, poor compliance was the most prevalent trigger of rehospitalization in our center. In contrast, clinician inertia might lead to suboptimal management of patients with HF. Since the Asian population has lower body weight and higher sensitivity to drugs than the Western population, underdosing and underprescription of HF-modifying drugs were common [23]. As generally known, suboptimal doses of ACE inhibitors, ARBs, beta-blockers, and aldosterone antagonists could subsequently increase the mortality and rehospitalization rate in patients with HF, particularly HFrEF.

The Cox regression model of six-month mortality was presented in Table 8 . From this study, the hazard ratios of tachycardia during admission and CKD were 1.938 and 2.165, respectively. Tachycardia on admission and CKD increase the risk of mortality at the six-month follow-up even though it is not statistically significant. It can as the effect of a smaller number of respondents compared to other studies. Assessment for tachycardia and CKD is needed in the management of a patient with increasing survival as the finding in this study showed shorter time survival in patients with tachycardia and CKD.

Tachycardia at admission and pre-existing CKD could be predictors for worse clinical outcomes in the next six months after discharge. Although these two variables were not statistically significant, which might be related to the insufficient number of respondents, the confidence interval indicated a tendency of higher death rate, as shown in the survival rates on the Kaplan Meier estimate. Higher heart rate during the acute event of HF results from neurohormonal disturbances. This neurohormonal activation acts as a mechanism to compensate for low cardiac output, which is beneficial in the short term [24]. However, in the long term, tachycardia might increase myocardial oxygen demand, induce additional ischemia, and cause arterial stiffness [25]. Most patients in this population had acute decompensation episodes from chronic HF; therefore, they might be previously treated with heart-rate-lowering agents, such as beta-blockers. In this situation, tachycardia at admission may reflect poor heart rate control in chronic HF. Eventually, worsened clinical outcomes in patients with AHF with tachycardia may result from the interaction between neurohormonal alteration, poor heart rate control, suboptimal previous treatment, and severity of the syndrome itself [25-28].

Several established studies have shown that CKD is associated with poor prognosis in both acute and chronic HF. The higher the blood urea and creatinine levels, the worse outcomes in patients admitted with AHF [29-33]. Renal worsening in patients with AHF also contributed to the need for ICU, respiratory failure requiring mechanical ventilation, and prolonged length of stay in the hospital [32]. Notably, CKD presents in $>40 \%$ of patients with HF [34]. CKD affects HF and vice versa, namely, cardiorenal syndrome to describe the relationship of these two [33]. The risk of cardiac impairment increases gradually with the decline in kidney 
function. In the long term, CKD causes fluid retention that further increases preload. Resistant hypertension, which is commonly encountered in advanced CKD, could increase the cardiac workload as a consequence of the elevated afterload [30]. In contrast, low cardiac output and congestion as the results of HF could cause hypoperfusion and renal vein congestion, leading to the deterioration of renal function [34]. Even though RAAS acts as a compensatory mechanism in HF, its benefits decline over time, even contributing to further cardiac remodeling which amplifies the clinical progression of HF. Long-term cardiac effects of RAAS are cardiac hypertrophy and fibrosis. Whereas the detrimental effects in the kidney are reduced medullary blood flow, tubular fibrosis, and efferent and afferent arterial vasoconstriction [30,32]. The result of this complex interaction may explain the worse outcome reported in patients with AHF with CKD compared to patients with AHF without pre-existing renal dysfunction.

This study has several limitations. First, the number of subjects was rather small. This unfavorable situation was related to a significant decrease in the number of patients visiting hospitals as the COVID-19 pandemic began in March 2020 in our region. Besides, in the same year, there was a cooperation withdrawal of the national insurance with our institution. This situation further limited the patient's preference to seek medical service to our center. Second, this evaluation came from a single-center experience and might not represent the characteristics and outcomes of HF in Indonesia's general population. Third, some parameters from the echocardiography examination were incompletely recorded. Fourth, natriuretic peptide and iron studies from the laboratory were only performed in a small number of patients, precluding further elaboration of these data. Lastly, our six-month follow-up did not include the type of medication with their doses. This situation contributed to the difficulty in evaluating whether the patients had received optimal medication after discharge. Hopefully, an upcoming larger representative nationwide registry could provide additional and valuable information regarding the clinical outcomes of the patients recently hospitalized with AHF.

\section{Conclusions}

This single-center and prospective study analyzed the six-month clinical outcomes following hospital discharge in patients with AHF who had predominantly HFrEF, were overweight or obese, had CAD and hypertension, and presented with ADHF. Poor patient compliance was considered the most common precipitating factor for rehospitalization. Although in-hospital mortality was relatively low, the six-month death and readmission rates were still high and worrisome. Tachycardia on admission and pre-existing CKD had been shown as potential predictors for the worse long-term adverse events in this population.

\section{Appendices}

\section{Contribution details}

Leonardo Paskah Suciadi: conceptualization, methodology, validation, investigation, resources, writingoriginal draft, writing-review and editing, supervision, and project administration

Kevin Wibawa: formal analysis, investigation, writing-original draft, writing-review and editing, and visualization

Giovanni Jessica: formal analysis, investigation, writing-original draft, and visualization

Joshua Henrina Sundjaja: formal analysis, investigation, and writing-review and editing

Irvan Cahyadi: formal analysis and investigation

Bryany Titi Santi: formal analysis and writing-review and editing

Titus Kurnia Hariadi: resources

Firman Tedjasukmana: resources

Nathania Marliani Kristanti: resources

Elisa Feriyanti Pakpahan: resources

Reynold Agustinus Manullang: resources

Antono Sutandar: resources and writing-review and editing

\section{Additional Information}

Disclosures 
Human subjects: Consent was obtained or waived by all participants in this study. Siloam Hospital Ethics Commitee issued approval 051/SHKJ/S/V/2021. We, the undersigned, herewith certify that: The institution has been informed regarding the observational research activities in hospitalized patients with heart failure at the Cardiology Department from October 2019 until January 2021 under the work of dr. Leonardo Paskah Suciadi and team. The research mechanisms have been directed to follow the standard operating protocols of our institution. The researcher team has been given permission and approval upon data collected and analysed at the cardiac intensive care unit, wards, and outpatient clinics. Animal subjects: All authors have confirmed that this study did not involve animal subjects or tissue. Conflicts of interest: In compliance with the ICMJE uniform disclosure form, all authors declare the following: Payment/services info: All authors have declared that no financial support was received from any organization for the submitted work. Financial relationships: All authors have declared that they have no financial relationships at present or within the previous three years with any organizations that might have an interest in the submitted work. Other relationships: All authors have declared that there are no other relationships or activities that could appear to have influenced the submitted work.

\section{References}

1. Snipelisky D, Chaudhry SP, Stewart GC: The many faces of heart failure . Card Electrophysiol Clin. 2019, 11:11-20. 10.1016/j.ccep.2018.11.001

2. Shah RU, Tsai V, Klein L, Heidenreich PA: Characteristics and outcomes of very elderly patients after first hospitalization for heart failure. Circ Heart Fail. 2011, 4:301-7. 10.1161/CIRCHEARTFAILURE.110.959114

3. Ponikowski P, Voors AA, Anker SD, et al.: 2016 ESC Guidelines for the diagnosis and treatment of acute and chronic heart failure: the task force for the diagnosis and treatment of acute and chronic heart failure of the European Society of Cardiology (ESC) Developed with the special contribution of the Heart Failure Association (HFA) of the ESC. Eur Heart J. 2016, 37:2129-200. 10.1093/eurheartj/ehw128

4. Storrow AB, Jenkins CA, Self WH, et al.: The burden of acute heart failure on U.S. emergency departments JACC Heart Fail. 2014, 2:269-77. 10.1016/j.jchf.2014.01.006

5. Filippatos G, Angermann CE, Cleland JGF, et al.: Global differences in characteristics, precipitants, and initial management of patients presenting with acute heart failure. JAMA Cardiol. 2020, 5:401-10. 10.1001/jamacardio.2019.5108

6. Ling HS, Chung BK, Chua PF, et al.: Acute decompensated heart failure in a non cardiology tertiary referral centre, Sarawak General Hospital (SGH-HF). BMC Cardiovasc Disord. 2020, 20:511. 10.1186/s12872-02001793-7

7. Siswanto BB, Radi B, Kalim H, et al.: Heart failure in NCVC Jakarta and 5 hospitals in Indonesia . CVD Prev Control. 2010, 5:35-8. 10.1016/j.cvdpc.2010.03.005

8. Savarese G, Lund LH: Global public health burden of heart failure . Card Fail Rev. 2017, 3:7-11. 10.15420/cfr.2016:25:2

9. Rajadurai J, Tse HF, Wang CH, Yang NI, Zhou J, Sim D: Understanding the epidemiology of heart failure to improve management practices: an Asia-Pacific perspective. J Card Fail. 2017, 23:327-39. 10.1016/i.cardfail.2017.01.004

10. Ferreira JP, Girerd N, Rossignol P, Zannad F: Geographic differences in heart failure trials . Eur J Heart Fail. 2015, 17:893-905. 10.1002/ejhf.326

11. Sato N: Epidemiology of heart failure in Asia. Heart Fail Clin. 2015, 11:573-9. 10.1016/j.hfc.2015.07.009

12. Forsyth P, Richardson J, Lowrie R: Patient-reported barriers to medication adherence in heart failure in Scotland. Int J Pharm Pract. 2019, 27:443-50. 10.1111/ijpp.12511

13. De Smedt RH, Haaijer-Ruskamp FM, Groenier KH, van der Meer K, Jaarsma T: Perceived adverse drug events in heart failure: patients' perception and related factors. J Cardiovasc Nurs. 2011, 26:250-60. 10.1097/JCN.0b013e318200ed94

14. Mor A, Thomsen RW, Ulrichsen SP, Sørensen HT: Chronic heart failure and risk of hospitalization with pneumonia: a population-based study. Eur J Intern Med. 2013, 24:349-53. 10.1016/i.ejim.2013.02.013

15. Corrales-Medina VF, Musher DM, Shachkina S, Chirinos JA: Acute pneumonia and the cardiovascular system. Lancet. 2013, 381:496-505. 10.1016/S0140-6736(12)61266-5

16. Bobylev AA, Rachina SA, Avdeev SN, et al.: Etiology of community-acquired pneumonia in patients with chronic heart failure. Pulmonologiya. 2019, 29:293-301. 10.18093/0869-0189-2019-29-3-293-301

17. Kadoglou NPE, Bracke F, Simmers T, Tsiodras S, Parissis J: Influenza infection and heart failure-vaccination may change heart failure prognosis?. Heart Fail Rev. 2017, 22:329-36. 10.1007/s10741-017-9614-7

18. Alon D, Stein GY, Korenfeld R, Fuchs S: Predictors and outcomes of infection-related hospital admissions of heart failure patients. PLoS One. 2013, 8:e72476. 10.1371/journal.pone.0072476

19. Gupta R, Testani J, Collins S: Diuretic resistance in heart failure. Curr Heart Fail Rep. 2019, 16:57-66. 10.1007/s11897-019-0424-1

20. Reyes EB, Ha JW, Firdaus I, et al.: Heart failure across Asia: same healthcare burden but differences in organization of care. Int J Cardiol. 2016, 223:163-7. 10.1016/j.ijcard.2016.07.256

21. Seth S, Khanal S, Ramakrishnan S, Gupta N, Bahl V: Epidemiology of acute decompensated heart failure in India: the AFAR study (acute failure registry study). J Pract Cardiovasc Sci. 2015, 1:1-99. 10.4103/23955414.157563

22. Cheraghi M, Sadeghi M, Sarrafzadegan N, Pourmoghadas A, Ramezani MA: Prognostic factors for survival at 6-month follow-up of hospitalized patients with decompensated congestive heart failure. ARYA Atheroscler. 2010, 6:112-7.

23. Teng T-HK, Tromp J, Tay WT, et al.: Prescribing patterns of evidence-based heart failure pharmacotherapy and outcomes in the ASIAN-HF registry: a cohort study. Lancet Glob Health. 2018, 6:1008-18. 10.1016/S2214-109X(18)30306-1

24. Hartupee J, Mann DL: Neurohormonal activation in heart failure with reduced ejection fraction . Nat Rev Cardiol. 2017, 14:30-8. 10.1038/nrcardio.2016.163 
25. Metra M: Tachycardia after a heart failure hospitalization: another piece of the puzzle? . JACC Heart Fail. 2013, 1:497-9. 10.1016/j.jchf.2013.10.003

26. Greene SJ, Vaduganathan M, Wilcox JE, et al.: The prognostic significance of heart rate in patients hospitalized for heart failure with reduced ejection fraction in sinus rhythm: insights from the EVEREST (efficacy of vasopressin antagonism in heart failure: outcome study with tolvaptan) trial. JACC Heart Fail. 2013, 1:488-96. 10.1016/j.jchf.2013.08.005

27. Ferrari R, Fox K: Heart rate reduction in coronary artery disease and heart failure . Nat Rev Cardiol. 2016, 13:493-501. 10.1038/nrcardio.2016.84

28. Kotecha D, Flather MD, Altman DG, et al.: Heart rate and rhythm and the benefit of beta-blockers in patients with heart failure. J Am Coll Cardiol. 2017, 69:2885-96. 10.1016/j.jacc.2017.04.001

29. Ruocco G, Palazzuoli A, Ter Maaten JM: The role of the kidney in acute and chronic heart failure . Heart Fail Rev. 2020, 25:107-18. 10.1007/s10741-019-09870-6

30. Grande D, Gioia MI, Terlizzese P, Iacoviello M: Heart failure and kidney disease . Adv Exp Med Biol. 2018, 1067:219-38. 10.1007/5584_2017_126

31. Lawson CA, Testani JM, Mamas M, Damman K, Jones PW, Teece L, Kadam UT: Chronic kidney disease, worsening renal function and outcomes in a heart failure community setting: a UK national study. Int J Cardiol. 2018, 267:120-7. 10.1016/j.ijcard.2018.04.090

32. Palazzuoli A, Lombardi C, Ruocco G, Padeletti M, Nuti R, Metra M, Ronco C: Chronic kidney disease and worsening renal function in acute heart failure: different phenotypes with similar prognostic impact?. Eur Heart J Acute Cardiovasc Care. 2016, 5:534-48. 10.1177/2048872615589511

33. Smith DH, Thorp ML, Gurwitz JH, et al.: Chronic kidney disease and outcomes in heart failure with preserved versus reduced ejection fraction: the Cardiovascular Research Network PRESERVE Study. Circ Cardiovasc Qual Outcomes. 2013, 6:333-42. 10.1161/CIRCOUTCOMES.113.000221

34. Shiba N, Shimokawa H: Chronic kidney disease and heart failure: bidirectional close link and common therapeutic goal. J Cardiol. 2011, 57:8-17. 10.1016/j.jjcc.2010.09.004 